\title{
Acute Cellular Allograft Nephropathy
}

National Cancer Institute

\section{Source}

National Cancer Institute. Acute Cellular Allograft Nephropathy. NCI Thesaurus. Code C114839.

A sudden onset of T-cell mediated immune response occurring after transplantation, directed against donor kidney alloantigens. 